\title{
Familial primary pigmented nodular adrenocortical disease without Carney complex (CNC): A case report and review of literature
}

\author{
Vaibhav Pandey ${ }^{1^{*}}$, Vivek Srivastava ${ }^{2}$, Anand kumar ${ }^{2}$, Mumtaz Ansari $^{2}$, S. K. Singh ${ }^{3}$ \\ ${ }^{1}$ Department of Paediatric Surgery, Institute of Medical Sciences, Banaras Hindu University, Varanasi, India; \\ *Corresponding Author: sunny.imsbhu@gmail.com \\ ${ }^{2}$ Department of General Surgery, Institute of Medical Sciences, Banaras Hindu University, Varanasi, India \\ ${ }^{3}$ Department of Endocrinology, Institute of Medical Sciences, Banaras Hindu University, Varanasi, India
}

Received 23 July 2013; revised 20 August 2013; accepted 19 September 2013

Copyright (C) 2013 Vaibhav Pandey et al. This is an open access article distributed under the Creative Commons Attribution License, which permits unrestricted use, distribution, and reproduction in any medium, provided the original work is properly cited.

\begin{abstract}
Primary pigmented nodular adrenocortical disease (PPNAD) is a rare cause of familial Cushing's syndrome. It is characterized by bilateral adrenocortical hyperplasia with small to normal-sized adrenal glands containing multiple small adrenal cortical pigmented nodules [1,2]. PPNAD may occur in an isolated form or as familial PPNAD. Familial cases of PPNAD are usually associated with Carney complex in which Cushing's syndrome is the most common endocrine manifestation [3]. Familial cases of PPNAD without associated Carney complex are very rare. Only a few cases of familial isolated PPNAD have been reported in the literature, mostly in females [4]. Isolated familial PPNAD has got a better prognosis than familial PPNAD associated with Carney Complex. This observation has important consequences for clinical management, follow-up and genetic counselling of such patients. Familial cases of PPNAD are rare and mostly present in females with associated Carney complex. We herein report a case of familial Cushing's syndrome in male siblings due to PPNAD without associated Carney complex.
\end{abstract}

Keywords: PPNAD; Carney Complex; Familial Cushing's Syndrome

\section{INTRODUCTION}

Primary pigmented nodular adrenocortical disease (PPNAD) is a form of bilateral adrenocortical hyperpla- sia that is often associated with corticotrophin (ACTH)independent Cushing's syndrome (CS) and is characterized by small to normal-sized adrenal glands containing multiple small cortical pigmented nodules [1,2]. PPNAD may occur in an isolated form or be associated with the multiple neoplasia syndrome, a complex of spotty skin pigmentation, myxomas, and endocrine overactivity, or Carney complex, in which Cushing's syndrome is the most common endocrine manifestation [3]. Although rare, familial cases of isolated PPNAD have been reported, most commonly in females [4]. We herein report a case of familial Cushing's syndrome in male siblings due to PPNAD without associated Carney complex.

\section{CASE REPORT}

A 13 years old male presented with complaints of progressive increase in body weight from the last 5 years, easy bruisability and recurrent infections. Further questioning revealed significant central abdominal weight gain over the preceding 6 months with proximal muscle weakness. On examination features suggestive of Cushing's syndrome were present like centripetal fat distribution with abdominal striae, proximal myopathy and skin atrophy with spontaneous bruises Figure 1. Blood pressure readings were mildly elevated. There was no visual field defect. He had normal secondary sexual characteristics and was clinically euthyroid. The baseline cortisol level was $22 \mu \mathrm{g} / \mathrm{dl}$ [8 AM] and there was failure of sup-pression with the $1 \mathrm{mg}$ overnight dexamethasone suppression test, with a post-suppression cortisol level of 42 $\mu \mathrm{g} / \mathrm{dl}$. The baseline adrenocorticotrophic hormone (ACTH) level was $24.3 \mathrm{pg} / \mathrm{ml}$. Endocrine evaluation for other secondary causes of osteoporosis revealed normal calcium profiles, thyroid function and testosterone level. 


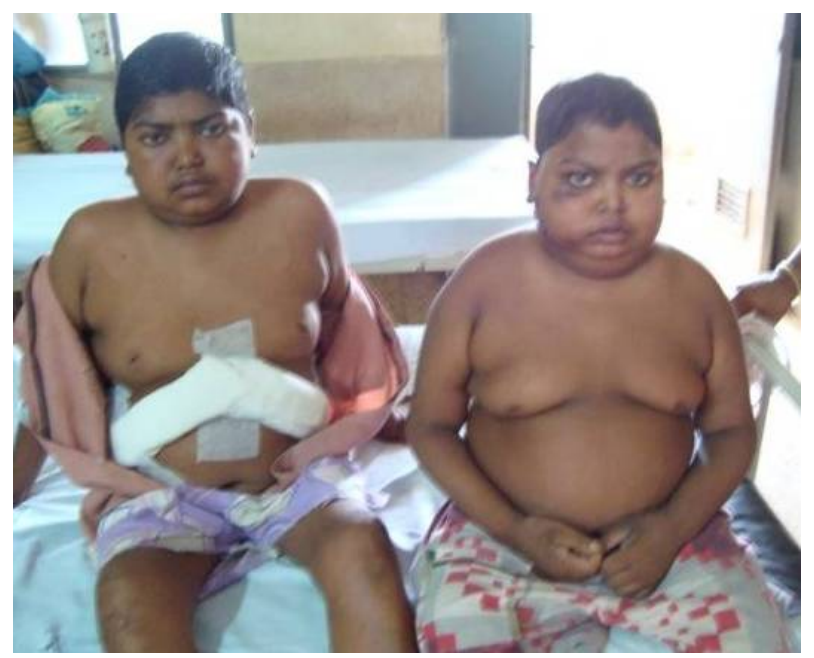

Figure 1. Siblings with features of Cushing's Syndrome.

The patient had similar clinical \& laboratory finding as his elder brother who was diagnosed as a case of PPNAD and was cured following bilateral adrenalectomy Figure 2.

So on the basis of the clinical and laboratory data in the background of a significant family history of the diagnosis of hypercortisolism due to familial PPNAD was made.

The patient underwent bilateral adrenalectomy through a bilateral subcostal approach. The diagnosis was then confirmed histopathologically Figure 3. Patient was discharged on oral steroids 10 - $15 \mathrm{mg} /$ day and Calcium and Vitamin D3 supplimentation. In the 6 months following adrenalectomy, there was resolution in his clinical features with less facial rounding and plethora. His weight decreased from 37 to $33 \mathrm{~kg}$ within 2 months and the central adiposity decreased. Features of CNC were absent in both the cases on subsequent regular follow up over a period of ten years.

\section{DISCUSSION}

Cushing syndrome is a symptom complex that reflects excessive tissue exposure to cortisol. It may be adrenocorticotrophic hormone (ACTH) dependent or independent. ACTH-independent processes account for approximately $15 \%$ to $20 \%$ of cases in adults and $15 \%$ in children $>7$ years old. Of these ACTH-independent causes, almost all are due to adrenal adenoma or carcinoma [5]. Micronodular adrenal hyperplasia (MAH), and its pigmented variant, primary pigmented nodular adrenocortical disease (PPNAD) are rare [6]. Our patient had clinically evident features of Cushing's syndrome, which exists in about $84 \%$ of patients with PPNAD. Approximately $10 \%$ have a paucity of symptoms and are diagnosed late; this group represents latent PPNAD. The

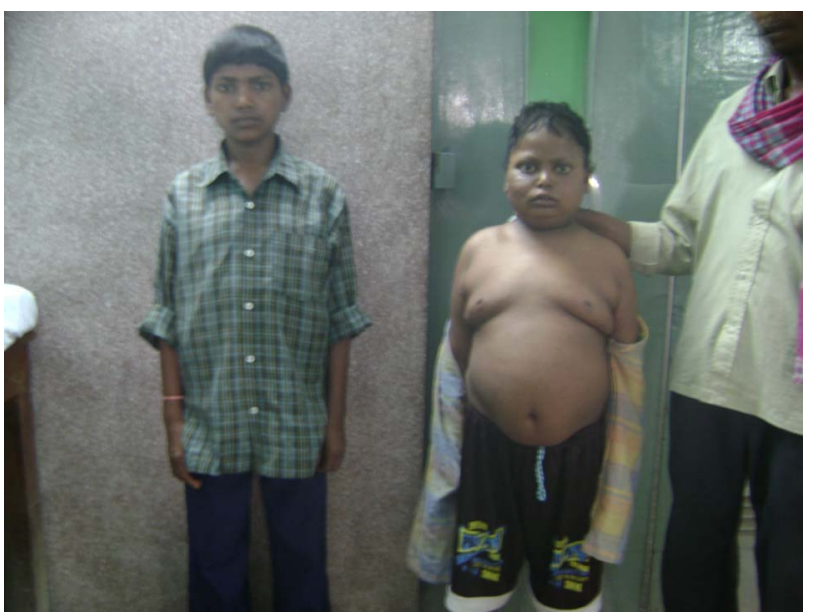

Figure 2. Elder brother in follow up after bilateral adrenalectomy.

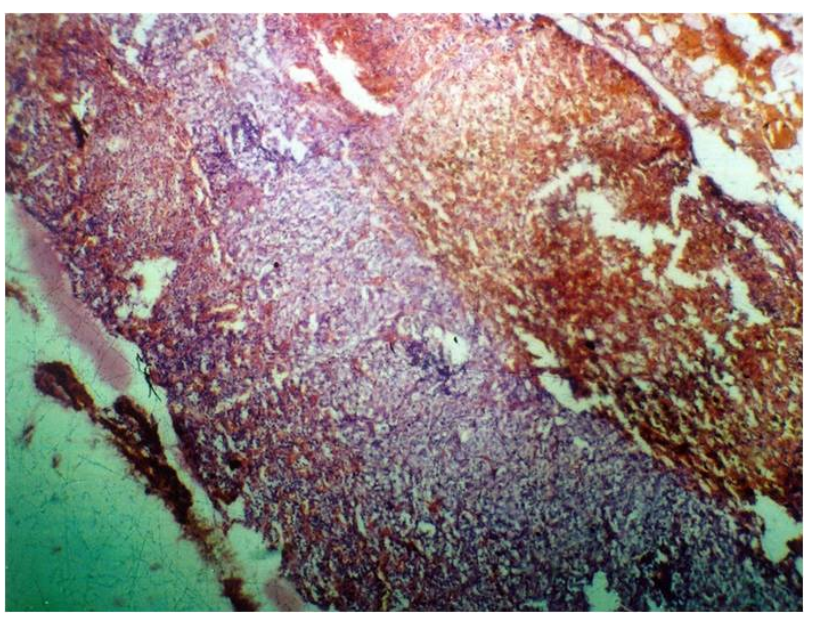

Figure 3. H\& E×400 Micronodules of size 2 - $4 \mathrm{~mm}$ and atrophic internodular area with brown/black pigmentation.

remaining $6 \%$ of patients have only biochemical evidence of Cushing's syndrome [7]. ACTH levels are usually low, normal or undetectable and adrenal glucocorticoid production is not suppressed by high-dose dexamethasone [8]. PPNAD forms part of a wider clinical spectrum of an autosomal dominant multiple endocrine neoplasia syndrome known as Carney complex characterised by complex of myxomas, spotty pigmentation and endocrine overactivity [9]. Half of PPNAD patients appear to be sporadic cases and the other half are familial, mostly associated with Carney complex (CNC) [2]. Familial cases of isolated PPNAD have also been reported and usually no other clinical features associated with CNC are seen in these families. A polypyrimidine tract mutation of the PRKAR1A gene has been described leading to a mild phenotype, almost exclusively CS due to PPNAD without any other associated features of CNC. The low penetrance of this genetic defect could explain the rarity of familial isolated PPNAD [4]. In PPNAD one 
or more macronodules ( $>1 \mathrm{~cm}$ in diameter) can be present unilaterally or bilaterally, making the differential diagnosis from ACTH independent macronodular adrenal hyperplasia (AIMAH) very difficult [10]. In patients with PPNAD there is delayed paradoxical increase of urinary free cortisol by $100 \%$ or more using the sequential LDDST and HDDST which forms the basis of Liddle's test, differentiating PPNAD from AIMNH [11]. In PPNAD, the characteristic macroscopic findings usually described include small brown-black nodules separated by an atrophic adrenal cortex. The cut surface is yellow, often with small brown foci. Histologically, the nodules consist of clear lipid-laden zona-fasciculata-type cells corresponding to the yellow areas on gross examination, while the brown foci consist of compact lipid-sparse zona-reticularis type cells [12]. Bilateral adrenalectomy is the treatment of choice for CS due to PPNAD. The laparoscopic approach is associated with a lower morbidity rate compared with the open technique, less postoperative pain, shorter hospitalization time and lower overall cost.

\section{CONCLUSION}

Isolated familial PPNAD is a rare entity and has got a better prognosis than familial PPNAD associated with Carney Complex. This observation has important consequences for clinical management, follow-up and genetic counselling of such patients.

\section{REFERENCES}

[1] Carney, J.A. and Young, W.F. (1992) Primary pigmented nodular adrenocortical disease and its associated conditions. Endocrinologist, 2, 6-21. http://dx.doi.org/10.1097/00019616-199201000-00003

[2] Stratakis, C.A. and Kirschner, L.S. (1998) Clinical and genetic analysis of primary bilateral adrenal diseases (micro- and macronodular disease) leading to Cushing syndrome. Hormone and Metabolic Research, 30, 456-63. http://dx.doi.org/10.1055/s-2007-978914

[3] Stratakis, C.A., Kirschner, L.S. and Carney, J.A. (2001) Clinical and molecular features of the Carney complex: Diagnostic criteria and recommendations for patient evaluation. The Journal of Clinical Endocrinology \& Metabolism, 86, 4041-4046.

http://dx.doi.org/10.1210/jc.86.9.4041

[4] Groussin, L., Horvath, A., Jullian, E., Boikos, S., ReneCorail, F., Lefebvre, H., et al. (2006) A PRKAR1A muta- tion associated with primary pigmented nodular adrenocortical disease in 12 kindreds. The Journal of Clinical Endocrinology \& Metabolism, 91, 1943-1949. http://dx.doi.org/10.1210/jc.2005-2708

[5] Arnaldi, G., Angeli, A., Atkinson, A.B., et al. (2003) Diagnosis and complications of Cushing's sy ndrome: A consensus statement. The Journal of Clinical Endocrinology \& Metabolism, 88, 5593-5602. http://dx.doi.org/10.1210/jc.2003-030871

[6] Stratakis, C.A. (2007) Adrenocortical tumors, primary pigmented adrenocortical disease (PPNAD)/Carney complex, and other bilateral hyperplasias: The NIH studies. Hormone and Metabolic Research, 39, 467-473. http://dx.doi.org/10.1055/s-2007-981477

[7] Carney, J.A., Gordon, H., Carpenter, P.C., Shenoy, B.V. and Go, V.L. (1985) The complex of myxomas, spotty pigmentation, and endocrine overactivity. Medicine, 64, 270-283.

http://dx.doi.org/10.1097/00005792-198507000-00007

[8] Sarlis, N.J., Chrousos, G.P., Doppman, J.L., Carney, J.A. and Stratakis, C.A. (1997) Primary pigmented nodular adrenocortical disease: Reevaluation of a patient with Carney complex 27 years after unilateral adrenalectomy. The Journal of Clinical Endocrinology \& Metabolism, 82, 1274-1278. http://dx.doi.org/10.1210/jc.82.4.1274

[9] Groussin, L., Jullian, E., Perlemoine, K., Louvel, A., Leheup, B., Luton, J.P., et al. (2002) Mutations of the PRKAR1A gene in Cushing's syndrome due to sporadic primary pigmented adrenocortical disease. The Journal of Clinical Endocrinology \& Metabolism, 87, 4324-4329. http://dx.doi.org/10.1210/jc.2002-020592

[10] Doppman, J.L., Travis, W.D., Nieman, L., Chrousos, G.P., Gomez, M.T., Cutler, G.B., et al. (1989) Cushing syndrome due to primary pigmented nodular adrenocortical disease: Findings at CT and MR imaging. Radiology, 172, 415-420.

[11] Stratakis, C.A., Sarlis, N., Kirschner, L.S., Carney, J.A., Doppman, J.L., Nieman, L.K., Chrousos, G.P. and Papanicolaou, D.A. (1999) Paradoxical response to dexamethasone in the diagnosis of primary pigmented nodular adrenocortical disease. Annals of Internal Medicine, 131, 585-591.

http://dx.doi.org/10.7326/0003-4819-131-8-199910190-0 $\underline{0006}$

[12] Neville, A.M., McGee, J.O’D., Isaacson, P.G. and Wright, N.A. (1992) Oxford Textbook of pathology. Vol. 2b. In: Pathology of systems. Oxford medical publications. Oxford University Press, New York, 1968-1986. 